\title{
FIR FILTER DESIGN BASED ON TOTAL LEAST SQUARES ERROR CRITERION
}

\author{
Soo-Chang Pei $\quad$ Chien-Cheng Tseng ${ }^{2}$ \\ ${ }^{1}$ Department of Electrical Engineering, National Taiwan University, Taipei, Taiwan, R. O. C \\ ${ }^{2}$ Department of Computer and Communication Engineering, National Kaohsiung First University of Technology and \\ Science, Kaohsiung, Taiwan, R.O.C.
}

\begin{abstract}
In this paper, an FIR filter design based on total least squares error criterion is investigated. The filter coefficients are obtained from the elements of the eigenvector corresponding to minimum eigenvalue of a real, symmetric and positive definite matrix. This design is not only optimal in total least squares error sense, it is also easy to incorporate linear constraints in time and frequency domain. Several design examples are used to illustrate the effectiveness of this new design approach.
\end{abstract}

\section{INTRODUCTION}

Conventionally, we often use the well-known McClellanParks-Rabiner (MPR) computer program and standard linear programming technique to design linear phase FIR filters according to the Chebyshev criterion which minimizes the maximum error in frequency response [1]. The minimax designs usually give the designers a smallest length filter for a given specification. However, MPR algorithm is difficult to incorporate both time and frequency domain constraint. And, linear programming technique needs a large memory space and considerable computation time. Thus, a number of researchers have considered linear phase FIR filter design using least-squares optimality criterion.

From literature survey, two well-documented least squares approaches for FIR filter designs are matrix inverse (IM) method and eigen-approach. The IM methods are based on solving a set of linear simultaneous equations by matrix inversion [2][3], and the eigen-approaches are based on the computation of an eigenvector of an appropriate real, symmetric, and positive-definite matrix [4][5]. Compared with minimax design, the advantage of least squares design is easy to add constraint and requires simple computation.

The purpose of this paper is to design linear phase FIR filters using total least squares (TLS) error criterion which has been successfully used to solve many engineering problems such as acoustic radiation problem, beamformer and harmonic retrieval etc [6]-[8]. The filter coefficients based on TLS criterion are obtained from the elements of the eigenvector corresponding to minimum eigenvalue of a real, symmetric and positive definite matrix. Due to this sake, the total least squares filter design is referred to as the new eigenfilter approach. The main difference between conventional and new eigenfilters is that conventional method needs to specify the reference point, but new approach does not require this choice. Moreover, the solution of new eigenfilter is closer to the solution of the IM method than the one of the conventional eigenfilter.

\section{FIR FILTER DESIGN BASED ON TOTAL LEAST SQUARES ERROR CRITERION}

2.1 Problem Statement:

A causal $N$ th order FIR filter can be represented as

$$
H(z)=\sum_{n=0}^{N} h(n) z^{-n}
$$

The magnitude responses of FIR filters is given by

$$
A(\omega)=\sum_{n=1}^{M} a_{n} \operatorname{trig}(\omega, n)
$$

where $\operatorname{trig}(\omega, n)$ is an approprite trigonometrical function [1]. The coefficient $a_{n}$ is related to the impulse response of the filter, whereas $M$ is a function of the filter order $N$. Defining the column vector

$$
\mathbf{a}=\left[\begin{array}{llll}
a_{1} & a_{2} & \cdots & a_{M}
\end{array}\right]^{t}
$$

and

$$
\mathbf{c}(\omega)=[\operatorname{trig}(\omega, 1) \operatorname{trig}(\omega, 2) \cdots \operatorname{trig}(\omega, M)]^{t}
$$

then we rewrite $\mathrm{eq}(2)$ as

$$
A(\omega)=\mathbf{a}^{t} \mathbf{c}(\omega)=\mathbf{c}^{t}(\omega) \mathbf{a}
$$

The notation $t$ denote the vector or matrix transpose. Now, the problem is that how we can find a such that the magnitude response $A(\omega)$ in eq(5) fit the desired magnitude response $D(\omega)$ as well as possible. Total least squares error measures will be studied in this paper.

2.2 TLS Design Method:

The well-known linear phase filter design problem is to find a filter weight a such that the desired magnitude response $D(\omega)$ is equal to the actual magnitude response $\mathbf{a}^{t} \mathbf{c}(\omega)$ of the filter, i.e.

$$
D(\omega)=\mathbf{a}^{t} \mathbf{c}(\omega)
$$

for each $\omega \in[0, \pi)$. In the space $\Re$ generated by $\mathbf{c}(\omega)$ and $D(\omega)$, it is clear that the expression $D(\omega)=\mathbf{a}^{t} \mathbf{c}(\omega)$ denotes a hyperplane. For a given frequency $\phi$, the $(\mathrm{c}(\phi), D(\phi))$ denotes a point in $\Re$. Now, the filter design problem can be restated as "we want the point $(\mathbf{c}(\phi), D(\phi))$ to fall on the hyperplane $D(\omega)=\mathbf{a}^{t} \mathbf{c}(\omega)$ for all $\phi \in[0, \pi)$ ". When the point $(\mathbf{c}(\phi), D(\phi))$ does not fall on the plane $D(\omega)=\mathrm{a}^{t} \mathrm{c}(\omega)$. the error between them can be measured in several ways. 
Two typical ones with geometric interpretation are shown in Fig.1. One error (type 1) is given by

$$
e(\phi)=\left|D(\phi)-\mathbf{a}^{t} \mathbf{c}(\phi)\right|
$$

the other (type 2) is given by

$$
e(\phi)=\frac{\left|D(\phi)-\mathbf{a}^{t} \mathbf{c}(\phi)\right|}{\sqrt{\mathbf{a}^{t} \mathbf{a}+1}}
$$

The least squares filter design problem means that the optimal filter weight $a$ is obtained by minimizing the squared errors

$$
J(\mathbf{a})=\int_{\omega \in R}|e(\omega)|^{2} d \omega
$$

where $R$ is the region $0 \leq \omega \leq \pi$, but excuding the transient band. Now, two types of least squares errors will be investigated in detail. Substitute eq(7) into eq(9), we obtain

$$
J(\mathbf{a})=\int_{\omega \in R}\left(D(\omega)-\mathbf{a}^{t} \mathbf{c}(\omega)\right)^{2} d \omega
$$

which is same as the least squares error measure of the conventional one [2]. Next, substitute eq(8) into eq(9), we have

$$
\begin{aligned}
J(\mathbf{a}) & =\int_{\omega \in R} \frac{\left(D(\omega)-\mathbf{a}^{t} \mathbf{c}(\omega)\right)^{2}}{\mathbf{a}^{t} \mathbf{a}+1} d \omega \\
& =\frac{\hat{\mathbf{a}}^{t} \mathbf{Q}_{n} \hat{\mathbf{a}}}{\hat{\mathbf{a}}^{t} \hat{\mathbf{a}}}
\end{aligned}
$$

where $\mathbf{a}$ and $\mathbf{Q}_{n}$ are given by

$$
\begin{aligned}
\hat{\mathbf{a}} & =\left[\begin{array}{ll}
\mathbf{a}^{t} & -1
\end{array}\right]^{t} \\
\hat{\mathbf{c}}(\omega) & =\left[\begin{array}{ll}
\mathbf{c}(\omega)^{t} & D(\omega)
\end{array}\right]^{t} \\
\mathbf{Q}_{n} & =\int_{\omega \in R} \hat{\mathbf{c}}(\omega) \hat{\mathbf{c}}(\omega)^{t} d \omega
\end{aligned}
$$

Based on Rayleigh's principle, the minimum of $J(\mathbf{a})$ occurs at the eigenvector of the matrix $\mathbf{Q}_{n}$ corresponding to the minimum eigenvalue. Note that the $\mathbf{Q}_{n}$ is also a real symmetric and positive definite matrix. Since the solution vector $\mathbf{a}$ is simply the minimum eigenvector of matrix $\mathbf{Q}_{n}$ we call this least squares design as new eigenfilter design. In the [6], the least squares error of type 2 is named as total least squares error, so we claim that the new eigenfilter is optimal in total least squares error sense. Now, we summarize the design procedure of new eigenfilter as follows:

Step 1: Compute the matrix $\mathbf{Q}_{n}$.

Step 2: Calculate the minimum eigenvector $\hat{\mathbf{a}}_{o}$ of the ma$\operatorname{trix} \mathbf{Q}_{n}$

Step 3: Normalize the solution vector $\hat{\mathbf{a}}_{0}$ to the form $\left[\begin{array}{ll}\mathbf{a}_{o}^{t} & -1\end{array}\right]^{t}$. The final desired solution $\mathbf{a}_{n}$ is equal to $\mathbf{a}_{\circ}$.

It is clear that this new eigenfilter does not need to specify the reference point which is a basic requirement of the conventional eigenfilter.

\subsection{Design Example:}

In the example, we will compare the performance of three least squares methods. This example is performed with the MATLAB Language in an IBM PC compatible computer by using the above design procedures.

Example 1: Lowpass filter design:
Consider the problem of designing a lowpass filter with the following desired amplitude response

$$
D(\omega)= \begin{cases}1, & 0 \leq \omega \leq \omega_{p} \\ 0, & \omega_{s} \leq \omega \leq \pi \\ \text { don't care, } & \omega_{p}<\omega<\omega_{s}\end{cases}
$$

There are four cases of FIR filters with exactly linear phase, but only two of these could be applied to design lowpass filters, that is, case 1 and case 2 [1]. Here, we only consider case 1 filter whose elements of matrix $Q_{t}$ and $p$ are given by

$$
\begin{aligned}
q_{t}(i, j) & =\int_{R} \cos ((i-1) \omega) \cos ((j-1) \omega) d \omega \\
p(i) & =\int_{R} \cos ((i-1) \omega) D(\omega) d \omega \quad 1 \leq i, j \leq M
\end{aligned}
$$

where the region $R=\left[0, \omega_{p}\right] \cup\left[\omega_{s}, \pi\right]$. Fig. 2 shows the magnitude responses of three least squares approaches with order $N=32, \omega_{p}=0.2 \pi$, and $\omega_{s}=0.3 \pi$. The reference point $\omega_{0}$ chosen in the conventional eigenfilter is $\frac{\omega_{p}}{2}$. From this result, it is clear that the specification are well satisfied for three least squares methods. However, the stopband attenuation of conventional eigenfilter is slightly worse than the proposed method, because the amplitude response at the reference frequency $\omega_{0}$ must be satisfied exactly for the conventional eigenfilter design. Moreover, the distances between optimal solutions are listed below:

$$
\begin{aligned}
\left|\mathbf{a}_{e f}-\mathbf{a}_{t}\right| & =0.005109 \\
\left|\mathbf{a}_{n}-\mathbf{a}_{t}\right| & =0.000267
\end{aligned}
$$

where $\mathbf{a}_{t}$ is the solution of IM method and $\mathbf{a}_{e f}$ is the one of conventional eigenfilter. Thus, the solution of the new eigenfilter is closer to the solution of the conventional least squares solution than the one of the conventional eigenfilter.

\section{FIR FILTER DESIGN WITH LINEAR CONSTRAINT}

The main advantage of the least squares approach is that various time and frequency constraints can be incorporated. In the linear case, the general form of the constraints can be stated as

$$
\mathbf{E a}=\mathbf{k}
$$

where $\mathbf{E}$ is a $L \times M$ matrix and $\mathbf{k}$ is a $L \times 1$ vector. Note that $L$ is the number of the linear constraints which is usually smaller than the number of coefficients $M$. Moreover, we assume $\mathbf{E}$ is a full rank matrix in order to avoid redundant constraints. For the conventional least squares filter design, the closed-form solution can be obtained by using the well-known Lagrange multiplier method [2]. Moreover, the procedure to find the solution of the conventional constrained eigenfilter design is slightly complicated, the detail can be found in [4]. As to the new eigenfilter design which is our main focus, the design problem becomes

$$
\begin{array}{rc}
\text { Minimize } & \frac{\hat{\mathbf{a}}^{t} \mathbf{Q}_{n} \hat{\mathbf{a}}}{\hat{\mathbf{a}}^{t} \hat{\mathbf{a}}} \\
\text { Subject to } & \mathbf{E a}=\mathbf{k}
\end{array}
$$

where $\hat{a}^{t}=\left[a^{t}-1\right]$. The basic idea of solving this problem is to rewrite the constraint $\mathbf{E a}=\mathbf{k}$ as the following form:

$$
\hat{\mathbf{E}} \mathbf{a}=0
$$


where $\hat{\mathbf{E}}=\left[\begin{array}{ll}\mathbf{E} & \mathbf{k}\end{array}\right]$. Then, the problem is reduced to

$$
\begin{array}{cl}
\text { Minimize } & \frac{\hat{\mathbf{a}}^{t} \mathbf{Q}_{n} \hat{\mathbf{a}}}{\hat{\mathbf{a}}^{t} \hat{\mathbf{a}}} \\
\text { Subject to } & \hat{\mathbf{E}} \mathbf{a}=\mathbf{0}
\end{array}
$$

The key step of our method is that "all the vector a satisfying constraint $\hat{\mathbf{E}} \mathbf{a}=\mathbf{0}$ can be expressed as $\hat{\mathbf{a}}=\mathbf{B w}$, where the columns of $\mathbf{B}$ form an orthonormal basis of the null space of matrx $\hat{\mathbf{E}}$ ". Based on full rank assumption of $\mathbf{E}$, we have that $\mathbf{B}$ is a $(M+1) \times(M+1-L)$ matrix and $\mathbf{w}$ is a $(M+1-L) \times 1$ vector. Due to orthonormal condition, we obtain $\mathbf{B}^{t} \mathbf{B}=\mathbf{I}$, where $\mathbf{I}$ is a $(M+1-L) \times(M+1-L)$ identity matrix. Thus, the problem described in eq(15) can be simplified as:

$$
\text { Minimize } \quad \frac{\mathbf{w}^{t} \mathbf{B}^{t} \mathbf{Q}_{n} \mathbf{B} \mathbf{w}}{\mathbf{w}^{t} \mathbf{w}}
$$

which is an unconstrained optimization problem. Hence, the optimal solution $\mathbf{w}_{o}$ of this simplified problem is only the minimum eigenvector of matrix $\mathbf{B}^{t} \mathbf{Q}_{n} \mathbf{B}$. Finally, the desired optimal solution $\hat{\mathbf{a}}_{o}$ is equal to $\mathbf{B w}_{0}$. Now, the remaining problem is how to find the orthonormal basis of null space of matrix $\hat{\mathbf{E}}$. The well-known singular value decomposition (SVD) and QR decomposition can help us to solve this problem.

Finally, we summarize the entire procedure of the proposed method as follows:

Step 1: Use SVD or QR decomposition to find the orthonormal basis of null space of matrix $\hat{\mathbf{E}}$ and construct the matrix B.

Step 2: Find the minimum eigenvector $\mathbf{w}_{0}$ of matrix $\mathbf{B}^{t} \mathbf{Q}_{n} \mathbf{B}$.

Step 3: Calculate the optimal solution $\hat{\mathbf{a}}_{o}=\mathbf{B w}_{o}$.

Step 4: Normalize the solution vector $\hat{\mathbf{a}}_{o}$ to the form

$\left[\mathbf{a}_{o}^{t}-1\right]^{t}$. The final desired solution is given by $\mathbf{a}_{o}$.

Note that the unconstrained eigenfilter design needs to find the minimum eigenvector of matrix $Q_{n}$ with size $(M+1) \times(M+1)$. However, new constrained eigenfilter design only requires to find the minimum eigenvector of matrix $\mathbf{B}^{t} \mathbf{Q}_{n} \mathbf{B}$ with size $(M+1-L) \times(M+1-L)$. Thus, constrained eigenfilter has less computation load in searching minimum eigenvector.

Example 2: Notch filter design:

In this example, we will use notch filter design to demonstrate the effectiveness of proposed design algorithm described in the above. The frequency response of an ideal notch filter has unit gain for all frequency except notch frequency in which gain is zero [2]. Here, the case 1 FIR filter of even order $N$ is used to design notch filter. Thus, the relation of the parameter in eq(2) is $M=\frac{N}{2}+1, \operatorname{trig}(\omega, n)=$ $\cos ((n-1) \omega), a_{1}=h\left(\frac{N}{2}\right)$, and

$$
a_{n}=2 h\left(\frac{N}{2}-n+1\right) \quad \text { for } n=2,3, \cdots, \frac{N}{2}+1
$$

Now, the optimal filter coefficient $a_{i}$ can be chosen such that the amplitude response $A(\omega)$ is as close as desired response $D(\omega)$ defined by

$$
D(\omega)=1 \quad \text { for all } \omega \in[0, \pi)
$$

Moreover, to obtain zero gain at notch frequency $\omega_{n}$ and control the null width, the following constraints are introduced:

$$
A\left(\omega_{n}\right)=0
$$

$$
\frac{\partial^{i} A\left(\omega_{n}\right)}{\partial \omega^{i}}=0 \quad \text { for } i=1, \cdots, L-1
$$

After some maniputation, it can be shown that the linear constraints can be written as standard form $\mathbf{E a}=\mathbf{0}$. Now, let us see some numerical examples. Fig.3(a) shows the magnitude response of a notch filter using proposed method for $\omega_{n}=0.5 \pi, N=32$, and various $L$. It is clear that the frequency response is well satisfactory. When constraint number $L$ increases, the notch width increases accordingly. For comparison, Fig.3(b) and (c) show the magnitude responses of a notch filter using the well-known Lagrange multiplier and linear programming methods for $N=32, \omega_{n}=0.5 \pi$, and $L=1$. Basically, the linear programming method is a minimax design and Lagrange multiplier approach is a conventional least squares error design. From this result, it is obvious that proposed method almost has the same frequency response as Lagrange multiplier method. This is due to both are belong to least squares error approaches. Compared with equal-ripple design using linear programming method, the new eigenfilter method has better frequency response fitting for all frequency band $\mathrm{ex}$ cept at the range around the notch frequency $\omega_{n}$.

\section{CONCLUSION}

In this paper, linear-phase FIR filter designs using total least squares error criterion have been investigated. This design method is easy to incorporate linear constraints in time and frequency domain. Several design examples have been used to illustrate the effectiveness of this new design approach. However, only linear phase FIR filters are considered here. Thus, it is interesting to study IIR filter designs based on total least squares error criterion. This topic will be investigated in the future.

\section{REFERENCES}

[1] A. V. Oppenheim and R. W. Shafer, Discrete-time Signal Processing, Prentice-Hall Englewood Cliffs, NJ, 1989.

[2] M. H. Er, "Designing notch filter with controlled null width," Signal Processing, vol.24, pp.319-329, Sep. 1991.

[3] M. Okuda, M. Ikehara and S. Takahashi, "Fast and stable least-squares approach for the design of linear phase FIR filters," IEEE Trans. Signal Processing, Vol.46, pp.1485-1493, June, 1998.

[4] T. Q. Nguyen, "The design of arbitrary FIR digital filters using the eigenfilter method," IEEE Trans. Signal Processing, vol.41, pp.1128-1139, Mar. 1993.

[5] S. C. Pei, C. C. Tseng and W. S. Yang, "FIR filter designs with linear constraints using eigenfilter approach ,"IEEE Trans. Circuits Syst. II, vol.45, pp.232-237, Feb. 1998.

[6] S. V. Huffel and J. Vandewalle, The Total Least Squares Problem: Computational Aspects and Analysis. SIAM, Philadelphia 1991

[7] S. V. Huffel, Recent Advances in Total Least Squares Techniques and Errors-in-variables Modeling. SIAM, Philadelphia 1997.

[8] D. Z. Feng, Z Bao and L. C. Jiao, "Total least mean squares algorithm," IEEE Trans. Signal Processing, vol.46, pp.2122-2130, Aug. 1998. 


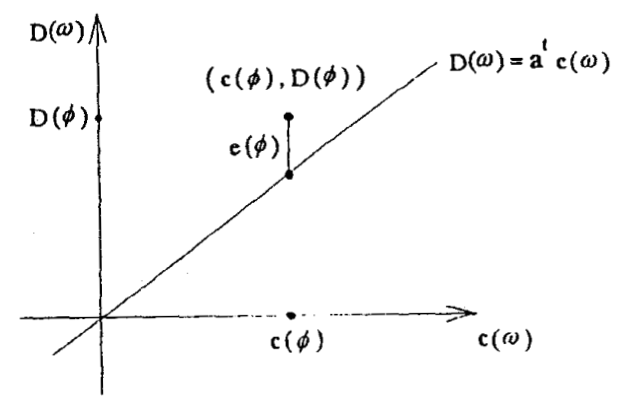

(a)

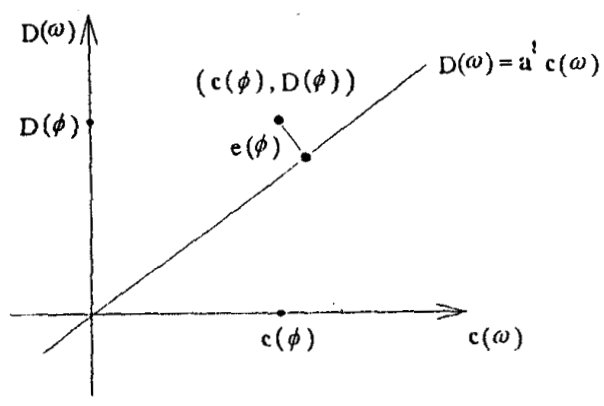

(b)

Fig.1 Geometric interpretation of two error measures at frequency $\omega=\phi(a)$ type 1 error (b) type 2 error.

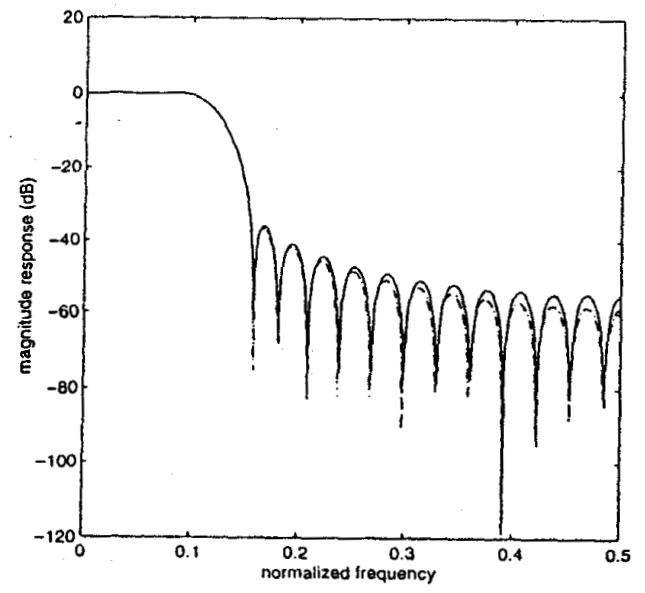

Fig.2 The magnitude response of a lowpass filter. The dashed line and dotted line almost overlap, conventional least squares design (dashed line), conventional eigenfilter design(solid line), total least squares design (dotted line).

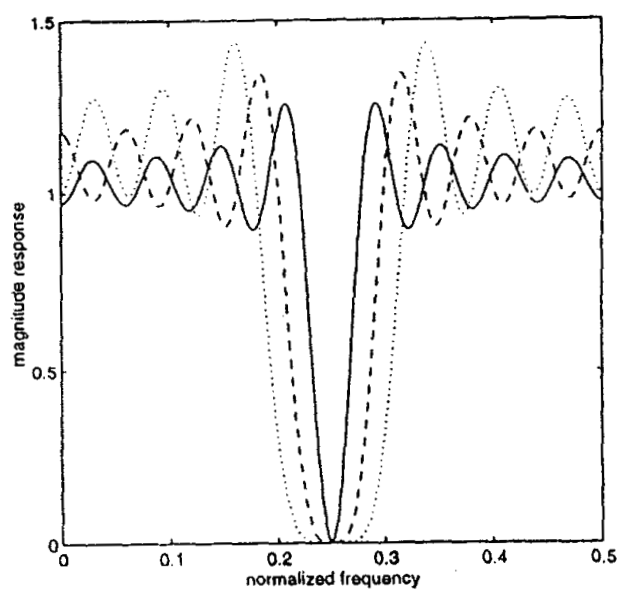

Fig.3(a) The magnitude response of a notch filter using TL$S$ method. $\mathrm{L}=1$ (solid line), $\mathrm{L}=3$ (dashed line), $\mathrm{L}=5$ (dotted line).

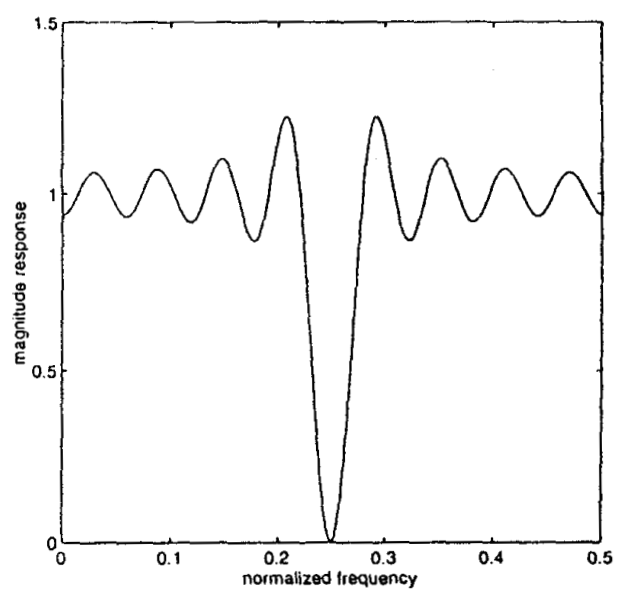

Fig.3(b) The magnitude response of a notch filter using Lagrange multiplier method for $\mathrm{L}=1$.

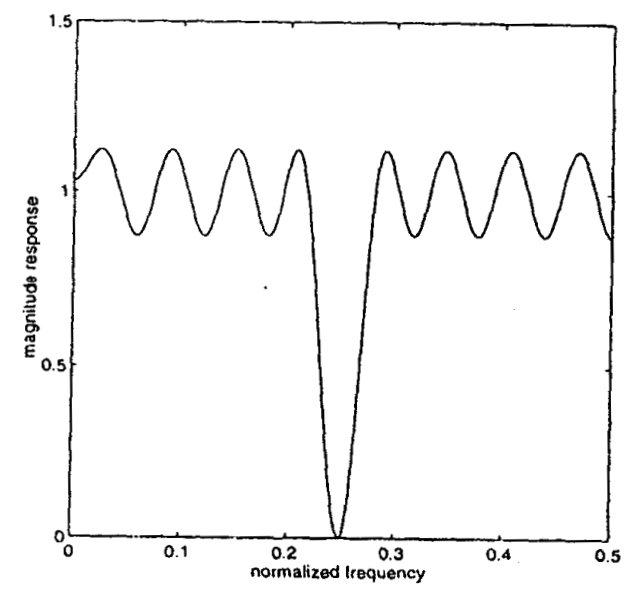

Fig.3(c) The magnitude response of a notch filter using linear programming method for $\mathrm{L}=1$. 